\title{
Transgenic Plasmodium parasites stably expressing Plasmodium vivax dihydrofolate reductase-thymidylate synthase as in vitro and in vivo models for antifolate screening
}

Voravuth Somsak ${ }^{1,2+}$, Chairat Uthaipibull ${ }^{1 *+}$, Parichat Prommana', Somdet Srichairatanakool ${ }^{2}$, Yongyuth Yuthavong ${ }^{1}$ and Sumalee Kamchonwongpaisan ${ }^{1}$

\begin{abstract}
Background: Plasmodium vivax is the most prevalent cause of human malaria in tropical regions outside the African continent. The lack of a routine continuous in vitro culture of this parasite makes it difficult to develop specific drugs for this disease. To facilitate the development of anti-P. vivax drugs, bacterial and yeast surrogate models expressing the validated $P$. vivax target dihydrofolate reductase-thymidylate synthase (DHFRTS) have been generated; however, they can only be used as primary screening models because of significant differences in enzyme expression level and in vivo drug metabolism between the surrogate models and $P$. vivax parasites.
\end{abstract}

Methods: Plasmodium falciparum and Plasmodium berghei parasites were transfected with DNA constructs bearing $P$. vivax dhfr-ts pyrimethamine sensitive (wild-type) and pyrimethamine resistant (mutant) alleles. Double crossover homologous recombination was used to replace the endogenous dhfr-ts of $P$. falciparum and $P$. berghei parasites with $P$. vivax homologous genes. The integration of Pvdhfr-ts genes via allelic replacement was verified by Southern analysis and the transgenic parasites lines validated as models by standard drug screening assays.

Results: Transgenic $P$. falciparum and $P$. berghei lines stably expressing PVDHFR-TS replacing the endogenous parasite DHFR-TS were obtained. Anti-malarial drug screening assays showed that transgenic parasites expressing wild-type PVDHFR-TS were pyrimethamine-sensitive, whereas transgenic parasites expressing mutant PVDHFR-TS were pyrimethamine-resistant. The growth and sensitivity to other types of anti-malarial drugs in the transgenic parasites were otherwise indistinguishable from the parental parasites.

Conclusion: With the permanent integration of Pvdhfr-ts gene in the genome, the transgenic Plasmodium lines expressing PVDHFR-TS are genetically stable and will be useful for screening anti-P. vivax compounds targeting PVDHFR-TS. A similar approach could be used to generate transgenic models specific for other targets of interest, thus facilitating the development of anti-P. vivax drugs in general.

\footnotetext{
* Correspondence: chairat@biotec.or.th

+ Contributed equally

'Protein-Ligand Engineering and Molecular Biology Laboratory, National

Center for Genetic Engineering and Biotechnology (BIOTEC), National Science and Technology Development Agency (NSTDA), Thailand Science

Park, Pathumthani 12120, Thailand

Full list of author information is available at the end of the article
} 


\section{Background}

Current anti-malarial drug development efforts are focused on screening for lead compounds against Plasmodium falciparum, the most lethal species. Plasmodium falciparum can be routinely cultured in vitro and is thus amenable to high throughput compound screening. Anti-malarial drug development against other human Plasmodium species, particularly Plasmodium vivax - which is the most prevalent cause of human malaria in tropical regions, with an estimated 80 million cases annually [1], is neglected in comparison, since continuous in vitro culture methods are not available for these species. The morbidity and mortality caused by $P$. vivax infection are greater than previously believed [2]. Chloroquine has been used as the standard treatment for blood stage vivax malaria for more than 40 years; however, chloroquine-resistant $P$. vivax has been reported in many parts of the world [3-5]. Therefore, there is an urgent need for new anti-vivax anti-malarial drugs.

One of the validated drug targets for the treatment of malaria infection is dihydrofolate reductase (DHFR; EC1.5.1.3), an essential enzyme for folate biosynthesis [6,7]. The efficacy of current anti-folate drugs targeting Plasmodium DHFR is compromised by mutations in the dhfr gene, which confer different levels of resistance to these drugs [8-10]. Point mutations in the P. vivax dhfr gene equivalent to antifolate resistance mutations found in P. falciparum have been associated with antifolate resistance in $P$. vivax in vitro [11-13], leading to the conclusion that wild-type $P$. vivax is sensitive to antifolates, and resistance develops through $d h f r$ mutations, similar to the case in P. falciparum.

Despite the emergence of drug-resistant dhfr mutants, DHFR-TS is still an attractive target for anti-malarial drug development owing to the availability of targetbased screening models and crystal structures of both $P$. falciparum [14] and $P$. vivax DHFR enzymes [15]. However, unlike $P$. falciparum, the development of antifolates directed against $P$. vivax has been hampered by the lack of a continuous in vitro parasite culture system. Clinical isolates can only be cultured for a short period of time $[16,17]$. Furthermore, mixed infection with other human malaria parasites can also complicate the drug screening results of clinical isolates. Recently, a $P$. vivax in vitro culture system using erythroblasts has been developed [18], but it is not practical for use in routine drug screening assay.

Surrogate cell expression systems expressing PvDHFR enzymes in yeast [19] and bacteria [20] have been developed as alternatives to parasite drug screening. However, these surrogate systems are of limited use since the level of target enzyme expression and mechanisms of drug metabolism differ markedly from Plasmodium parasites. A physiologically similar Plasmodium species is clearly a more attractive $P$. vivax surrogate, and this has been proven using transfection technology in which P. falciparum parasites expressing mutant $P v$ DHFR-TS have been shown to recapitulate the mutant PvDHFR-TS antifolate resistance [21]. In the same manner, transgenic P. falciparum parasites episomally expressing drug resistant $P v$ DHFR-TS mutant enzymes have also been developed for studying $P$. vivax drug resistance. However, these transgenic $P$. falciparum parasites still express endogenous PfDHFR-TS as well as episomally expressed PvDHFR-TS enzymes [22], which could confound drug screening results.

In order to facilitate antifolate screening specifically against PvDHFR-TS, transgenic P. falciparum and Plasmodium berghei parasites stably expressing PvDHFR-TS enzyme replacing the endogenous $P f$ or $P b D H F R-T S$ were generated. These transgenic lines were evaluated as in vitro and in vivo Plasmodium models for direct assessment of the antifolate efficacy against PvDHFRTS. These Plasmodium surrogate models were thus validated as alternative tools for screening PvDHFR-TS-targeted compounds.

\section{Methods \\ Parasites}

Plasmodium falciparum strains TM4/8.2 (wild-type DHFR) and K1CB1 (mutant DHFR at residues C59R + S108N) were gifts from Sodsri Thaithong, Faculty of Science, Chulalongkorn University, Thailand. Pyrimethamine-resistant strain K1CB1 was used as parental parasite to generate an in vitro $P$. vivax screening model. The parasites were maintained continuously in human erythrocytes at $37^{\circ} \mathrm{C}$ under $5 \% \mathrm{CO}_{2}, 1 \% \mathrm{O}_{2}$ and 94\% $\mathrm{N}_{2}$ in RPMI-1640 culture media supplemented with $24 \mathrm{mM} \mathrm{NaHCO} 3,40 \mu \mathrm{g} / \mathrm{mL}$ gentamicin, $25 \mathrm{mM}$ HEPES and $10 \%$ human serum [23]. For generation of in vivo screening models, the transgenic $P$. berghei parasite line MRA-867 (PbGFP), a gift from Chris Janse and Andy Waters at Leiden University Medical Center, the Netherlands, was used. This line contains the $g f p$ gene stably integrated as a single copy by double cross-over recombination into the $230 p$ locus, and does not contain a drug selectable marker gene $[24,25]$. Frozen parasites from stock were mechanically passaged at least once through female BALB/c mice before experiments. Animals were bled from the heart and parasitaemia measured by Giemsa-stained thin blood smear. Infected erythrocytes were suspended in PBS and infection for experiments was carried out by intra-peritoneal injection of approximately $1 \times 10^{7}$ parasitized erythrocytes. 


\section{Experimental animals}

Pathogen-free, six-week-old female BALB/c mice weighing 20-30 g were obtained from the National Laboratory Animal Center, Mahidol University, Thailand. They were kept for at least one week with tap water and pellet diet (CP diet 082, Perfect Companion Company, Bangkok, Thailand) ad libitum at $22-25^{\circ} \mathrm{C}$ and a 12-hour light/12-hour dark cycle. Experiments were started in seven- to eight-week-old animals. Animal experiments were ratified by the Ethical Committee on Animal Experimentation, Faculty of Medicine, Chiang Mai University, Chiang Mai, Thailand and by the Ethical Committee on Animal Experimentation, National Center for Genetic Engineering and Biotechnology (BIOTEC), Thailand. Animal experiments conformed to international and national guidelines for ethical conducts on the care and humane use of animals.

\section{Construction of transfection plasmids}

Wild-type and mutant $P$. vivax dhfr-ts genes were kindly given by Ubolsree Leartsakulpanich, BIOTEC, Thailand [11]. For generation of transgenic P. falciparum parasite stably expressing PvDHFR-TS, a transfection plasmid was constructed containing three expression cassettes: 1) blasticidin-S deaminase ( $b s d$ ) as a positive selection marker under the control of 5 ' flanking region of $P$. falciparum camodulin gene (Pfcam) and 3' UTR of P. falciparum histidine rich protein 2 (Pfhrp2) [26], 2) A fusion gene of cytosine deaminase and uracil phosphoribosyl transferase ( $y f c u)$ from Saccharomyces cerevisiae as a negative selection marker under the control of 5' flanking region of $P$. falciparum heat shock protein 86 gene (Pfhsp86) and 3' UTR of P. berghei dihydrofolate reductase (Pbdhfr) [27], and 3) The wild-type $d h f r$-ts gene of $P$. vivax under the control of 5' flanking region of $P f d h f r$ and 3' UTR of Pfhrp2 (Figure 1A). The 5' flanking region and truncated $5^{\prime}$ coding sequence of $P f d h f r-t s$ are the sites for homologous recombination and replacement of Pfdhfr-ts with Pvdhfr-ts gene in the P. falciparum parasite chromosome 4 .

Plasmodium berghei transfection plasmids were constructed from plasmids pL0017 [24] and pL0035 [28], which were kindly provided by Chris Janse and Andy Waters (Leiden University Medical Center, the Netherlands). In order to generate a $P$. berghei parasite line stably expressing wild-type PvDHFR-TS enzyme, transfection plasmids were modified to consist of two expression cassettes (Figure 1B). The first cassette has the wild-type Pvdhfr-ts gene flanked by 5' and 3'UTR Pbdhfr-ts gene sequences. The second cassette is a drug selection cassette containing a fusion gene of positive (human dihydrofolate reductase; hdhfr) and negative (yeast $y f c u$ ) selectable markers under the control of 5' flanking region of $P$. berghei elongation factor $1 \alpha-a$ (Pbef1 $\alpha-a)$ and 3'UTR sequence of Pbdhfr-ts gene.

For generation of $P$. berghei parasite stably expressing double mutant (S58R+S117N; SP21) PvDHFR-TS enzyme, the transfection plasmid contains the double mutant Pvdhfr-ts gene (Pvsp21) flanked by the Pbdhfr-ts gene 5' and 3' flanking sequences (Figure $1 \mathrm{C}$ ). The Pbdhfr-ts gene sequences serve as the sites for doublecrossover homologous recombination. The pyrimethamine-resistant $P v s p 21$ gene itself serves as a positive drug selectable marker for integration and gene replacement.

\section{Generation of transgenic parasite lines stably expressing PvDHFR-TS}

\section{Plasmodium falciparum transfection}

Transfection of $P$. falciparum parasite was performed following a standard protocol [29]. Briefly, synchronized ring stage $P$. falciparum parasite strain K1CB1 (pyrimethamine-resistant) was transfected with $100 \mu \mathrm{g}$ of transfection plasmid by electroporation $(0.2 \mathrm{~cm}$-gap electroporation cuvette, $0.310 \mathrm{kV}, 950 \mu \mathrm{F}$ ). The transgenic parasites were first selected with blasticidin. Once blasticidin-resistant parasites were obtained, they were further treated with the negative selectable drug 5-fluorocytosine (5-FC) to select for double crossover integration of Pvdhfr-ts gene replacing the endogenous Pfdhfrts gene. After several rounds of drug cycling, stable transgenic parasites with the desired integration event were obtained and cloned by limiting dilution.

\section{Plasmodium berghei transfection}

Transfection of $P$. berghei parasites was performed using an Amaxa Nucleofector device (Amaxa), following the standard protocol [30]. Purified schizonts of GFPexpressing $P$. berghei ANKA parasite line MRA-867 (PbGFP) were transfected with $10 \mu \mathrm{g}$ of linearized transfection plasmid in $100 \mu \mathrm{l}$ of Human T Cell Nucleofector solution (Amaxa) using programme U-33, and then injected intravenously into naïve $\mathrm{BALB} / \mathrm{c}$ recipient mice. Twenty-four hours post-injection, blood smears were made from the host animals and the presence of parasites checked by Giemsa staining. Drug-resistant parasites were selected by pyrimethamine administered to the drinking water $(70 \mu \mathrm{g} / \mathrm{ml})$. Under this selection regimen, PvDHFR-TS SP21 conferred pyrimethamine resistance to transgenic parasites. For selection of $P$. berghei harbouring wild-type $P v d h f r$-ts, after pyrimethamine selection for the positive drug marker $h d h f r$, infected mice were injected intra-peritoneally twice a day with 5FC (dissolved in $0.9 \% \mathrm{NaCl}$ ) at $1 \mathrm{~g} / \mathrm{kg}$ bodyweight for a period of three days to select for parasites in which the drug selectable markers had been excised by single homologous recombination via the Pbdhfr-ts 3'UTR repeated sequence elements [28], whilst retaining the 


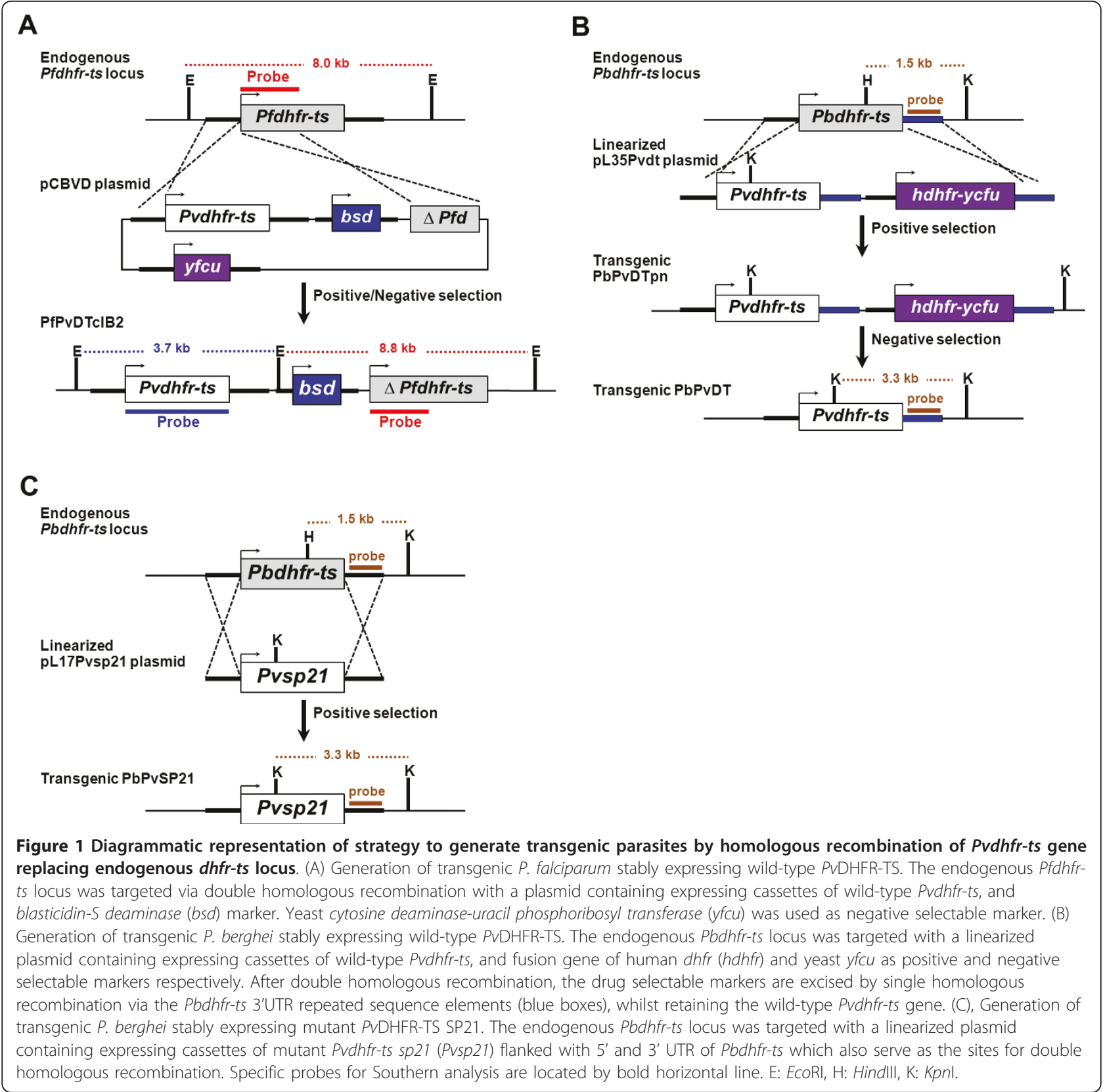

wild-type $P v d h f r$-ts gene. The PvDHFR-TS-expressing transgenic $P$. berghei parasites were subsequently cloned by the method of limiting dilution [31].

\section{Characterization of transgenic parasites}

Correct integration of the constructs into the host's endogenous $d h f r$-ts locus was demonstrated by Southern analysis of restricted genomic DNA from transgenic parasites. Genomic DNA from erythrocytes infected with transgenic $P$. falciparum harbouring $P v d h f r$-ts gene was purified using a QIAamp Blood Mini Kit (Qiagen) according to the manufacturer's instructions. Blood from mice infected with transgenic $P$. berghei harbouring Pvdhfr-ts or Pvsp 21 genes was collected by cardiac puncture, and then depleted of leucocytes by passing through a CF11 cellulose column (Whatman). Following lysis of the erythrocyte pellet with $0.2 \%(\mathrm{w} / \mathrm{v})$ saponin in PBS, total genomic DNA from parasites was obtained using a QIAamp Blood Mini Kit (Qiagen). The resulting DNA was then used for Southern analysis to confirm allelic replacement of the endogenous $d h f r-t s$ with the $P$. vivax homologue. Briefly, $30 \mu \mathrm{g}$ of genomic DNA from transgenic parasites was restriction-digested, separated in $0.6 \%(\mathrm{w} / \mathrm{v})$ agarose gel and transferred onto a Hybond 
$\mathrm{N}+$ nylon membrane (GE Healthcare) by capillary blotting. Digested DNA was fixed to the membrane by UV cross-linking and hybridized with specific probes prepared with a DIG labelling kit (Roche Applied Science). All probes used in this study are shown schematically in Figure 1. The pattern of hybridization was detected using alkaline phosphatase-conjugated anti-DIG antibody and CSPD reagent according the manufacturer's instructions (Roche Applied Science).

\section{Validation of transgenic parasites as models for PVDHFR- TS screening SYBR Green I assay}

Plasmodium falciparum strains TM4/8.2, K1CB1 and transgenic $P$. falciparum expressing PvDHFR-TS were maintained in human erythrocytes as previously described and synchronized to $1 \%$ ring stages at $2 \%$ haematocrit. Ninety microliters of cultured parasites were transferred to each well of a 96-well microtitre plate before $10 \mu \mathrm{l}$ of pyrimethamine (Sigma), chloroquine (Sigma) and dihydroartemisinin (a gift from Dafra Pharma International) at different concentrations were added in triplicate. The mixtures were incubated at $37^{\circ} \mathrm{C}$ under $5 \% \mathrm{CO}_{2}, 1 \% \mathrm{O}_{2}$ and $94 \% \mathrm{~N}_{2}$ for 48 hours. To determine parasite growth, $100 \mu \mathrm{l}$ of SYBR green I (at 1:5,000 dilution in culture media; Invitrogen) was added to the culture mixtures for 30 minutes at room temperature in the dark [32]. The SYBR green I-stained parasitized erythrocytes were then analysed using a Cytomics 500 FC MPL flow cytometer (Beckman Coulter) equipped with a 96-well plate adaptor. Erythrocytes were identified on the basis of their specific forward (FSC) and side (SSC) light-scattering properties. The green fluorescence signal was excited with an argon ion laser at a wavelength of $488 \mathrm{~nm}$ and the emission of the green fluorescence was detected using a 530/30 nm band pass filter. A previous analysis of non-infected erythrocytes was performed to determine a cut off for these cells to distinguish them from parasitized cells. The fluorescent intensity of a total of 100,000 events per sample was measured and data analysis was subsequently performed using CXP software (Beckman Coulter). For calculation of the percentage of parasite growth, the mean parasitaemia of untreated parasites was set at $100 \%$ growth. The concentration of drugs that inhibits $50 \%$ of the parasite growth $\left(\mathrm{IC}_{50}\right)$ was determined from the sigmoidal curve obtained by plotting the percentages of parasite growth against drug concentrations.

\section{GFP assay}

In order to validate transgenic $P$. berghei parasite stably expressing P $v$ DHFR-TS enzymes as in vivo models for antifolate drug screening, the assessment of anti-malarial drug efficacy in vivo was performed using the standard four-day suppressive test [33]. Groups of at least three mice were inoculated with approximately $1 \times 10^{7}$ parasitized erythrocytes intravenously and then treated with standard anti-malarials: pyrimethamine (Sigma), chloroquine (Sigma) or artesunate (Mekophar Chemical Pharmaceutical) once daily for four successive days from the day of parasite inoculation. The drugs at chosen doses (mg/kg of body weight) were freshly prepared in $100 \%$ DMSO (pyrimethamine and artesunate) or distilled water (chloroquine) and administered orally by gavage. It was found that pyrimethamine at $50 \mathrm{mg} / \mathrm{kg}$ was lethal to mice as all animals died soon after treatment with this dose by oral administration (data not shown). Hence, the maximum pyrimethamine dose was $30 \mathrm{mg} /$ $\mathrm{kg}$. Untreated controls were given either distilled water or DMSO only. The course of parasitaemia in untreated mice (control group) and in mice treated with different doses of drugs was monitored by flow cytometry since these parasites express GFP as fluorescence marker. A drop of blood was collected directly into wells of a 96well microtitre plate containing $200 \mu \mathrm{l}$ of RPMI-1640 culture media and heparin $(1 \mathrm{U} / \mu \mathrm{l})$. The GFP signal in these samples was measured as described above. For calculation of the growth inhibitory curves, the mean parasitaemia of the control samples was set at $0 \%$ inhibition. The concentration of drugs that inhibits $50 \%$ of the parasite growth $\left(E D_{50}\right)$ was determined from the sigmoidal curve obtained by plotting the percent parasite growth inhibition against drug concentrations.

\section{Statistical analysis}

Parasites' growth and growth inhibitory curves and statistical analysis of the data were performed using the GraphPad Prism software version 5.0 (GraphPad Software). The non-linear regression function for sigmoidal dose-response (variable slope) was used to calculate the best-fit $50 \%$ inhibition concentration $\left(\mathrm{IC}_{50}\right)$ or $50 \%$ effective concentration $\left(\mathrm{EC}_{50}\right)$ values.

\section{Results}

Generation of transgenic Plasmodium falciparum harbouring Pvdhfr-ts gene

Pyrimethamine-resistant $P$. falciparum strain K1CB1 was transfected with plasmid DNA for replacement of the endogenous $P f d h f r$-ts with wild-type Pvdhfr-ts. Following rounds of positive and negative selections, transgenic $P$. falciparum were obtained. A clonal parasite line designated PfPvDTclB2 was obtained and gene replacement verified by Southern blotting analysis. As shown in Figure 2A, hybridization with a $P v d h f r-t s$ DNA probe showed the expected $3.7 \mathrm{~kb}$ and $5.9 \mathrm{~kb}$ EcoRI-digested fragments from transgenic PfPvDTclB2 genomic DNA and control plasmid DNA, respectively, confirming the integration of $P v d h f r-t s$ at the correct site, while no 

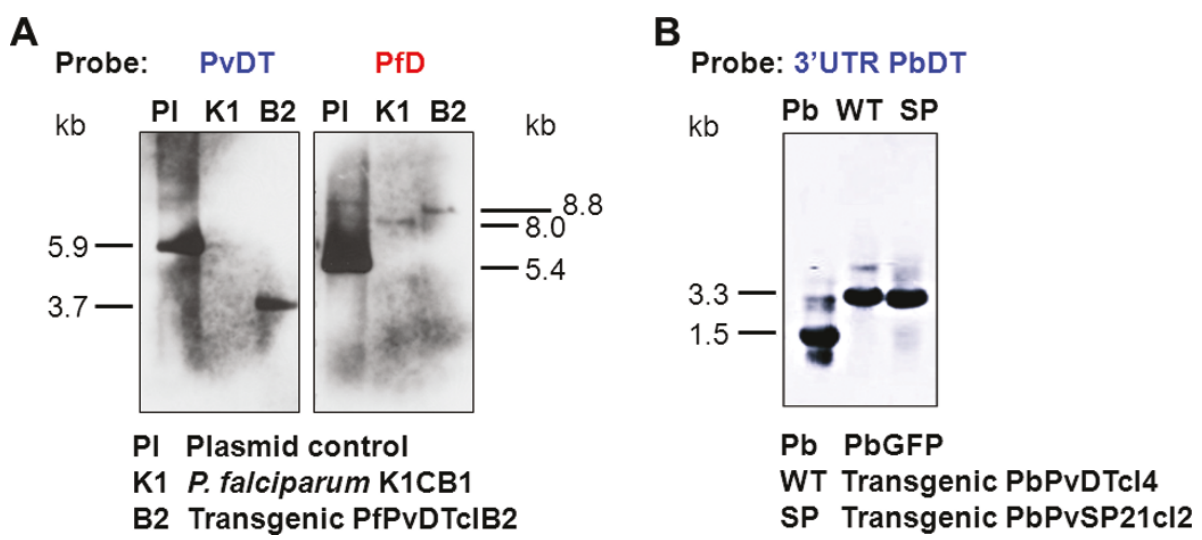

Figure 2 Southern analysis of transgenic parasites to confirm allelic replacement of dhfr-ts of Plasmodium falciparum (A) and Plasmodium berghei (B) with dhfr-ts gene from P. vivax. DNA probes specific to Pvdhfr-ts (PvDT), Pfdhfr (PfD) and $3^{\prime} U T R$ of Pbdhfr-ts ( $3^{\prime} U T R$ $\mathrm{PbDT})$ were used to detect restriction-digested fragments from genomic DNA of transgenic parasites. PI: pCBVD plasmid control; K1: $P$. falciparum K1CB1; B2: Transgenic PfPvDTcIB2; PbGFP: P. berghei GFP; WT: transgenic PbPvDTcl4; SP: transgenic PbPvSP21cl2.

signal was detected for parental P. falciparum K1CB1 line. Another DNA probe specific to Pfdhfr could detect the expected $8.8 \mathrm{~kb}, 8.0 \mathrm{~kb}$ and $5.4 \mathrm{~kb}$ EcoRI-digested fragments from genomic DNA of transgenic PfPvDTclB2, parental P. falciparum K1CB1 line and control plasmid DNA, respectively. From these results, it can be concluded that transgenic PfPvDTclB2 parasites stably express wild-type Pvdhfr-ts instead of endogenous $P f d h f r$-ts; furthermore, these parasites are clonal, lack episomal plasmid DNA and can be stably maintained without drug selection.

\section{Generation of transgenic Plasmodium berghei harbouring Pvdhfr-ts gene}

Blood stages of the reference GFP-expressing $P$. berghei parasite line PbGFP were transfected with the linear form of the transfection vectors by electroporation in order to introduce either wild-type Pvdhfr-ts or mutant Pvsp21 genes replacing the endogenous Pbdhfr-ts gene. For selection of $P$. berghei harbouring wild-type Pvdhfr$t s$, a two-step drug selection procedure was performed. First, transgenic parasites with integrated wild-type $P v d h f r$-ts were selected with pyrimethamine. Subsequently, negative selection was performed to select for parasites which had excised the positive-negative drug selection cassette in order to obtain parasites that have only wild-type $P v d h f r$-ts gene integrated to the $P$. berghei genome (Figure 1B). Transgenic $P$. berghei parasite clones stably expressing wild-type PvDHFR-TS (designated PbPvDTcl4) or double mutant PvDHFR-TS SP21 (designated $\mathrm{PbPvSP} 21 \mathrm{cl} 2$ ) were obtained and analysed by Southern blotting. As shown in Figure 2B, the KpnI/ HindIII digested genomic DNA fragments corresponding to the parental wild type and transgenic $P$. berghei parasites (1.5 kb and $3.3 \mathrm{~kb}$, respectively) were detected by a DNA probe specific to 3'UTR Pbdhfr-ts. Thus, this confirmed the successful generation of transgenic $P$. berghei parasites stably harbouring wild-type $P v d h f r$-ts or mutant Pvsp21, replacing the endogenous Pbdhfr-ts gene.

Drug sensitivity analysis of transgenic Plasmodium falciparum stably expressing wild-type PvDHFR-TS enzyme

Transgenic PfPvDTclB2 parasite was evaluated against standard anti-malarial drugs. Pyrimethamine is the standard antifolate drug and used as the primary compound to validate this system. As shown in Figure 3A and Table 1, the transgenic PfPvDTclB2 parasite was much more sensitive to pyrimethamine than the parental K1CB1 line, verifying that the wild-type Pvdhfr-ts gene replacing the $P f d h f r$-ts gene is a pyrimethamine-sensitive variant. Moreover, the level of pyrimethamine sensitivity in the transgenic PfPvDTclB2 parasite is the same as the antifolate-sensitive $P$. falciparum $\mathrm{TM} 4 / 8.2$ strain $\left(\mathrm{IC}_{50}=\right.$ $0.03 \pm 0.02 \mu \mathrm{M})$. The parental $\mathrm{K} 1 \mathrm{CB} 1$ line is also resistant to chloroquine, a 4-aminoquinoline drug that inhibits haemozoin formation in the food vacuole of the parasites. This transgenic PfPvDTclB2 line shows the same chloroquine-resistant phenotype as the parental $P$. falciparum $\mathrm{K} 1 \mathrm{CB} 1$ strain, with $\mathrm{IC}_{50}$ values of $49.5 \pm 5.8$ $\mathrm{nM}$ and $46.0 \pm 3.1 \mathrm{nM}$ respectively (Figure $3 \mathrm{~B}$ and Table 1), indicating that the $d h f r$-ts gene replacement did not affect sensitivity to drugs not targeting DHFRTS. Another non-antifolate drug control used in this study was dihydroartemisinin (DHA). All parasites tested in this study were sensitive to $\mathrm{DHA}$ at the $\mathrm{IC}_{50}$ values of $0.6 \pm 0.1 \mathrm{nM}, 0.7 \pm 0.3 \mathrm{nM}$ and $0.4 \pm 0.1 \mathrm{nM}$ for $P$. falciparum TM4/8.2, K1CB1 and transgenic PfPvDTclB2, respectively (Figure $3 \mathrm{C}$ and Table 1 ). 

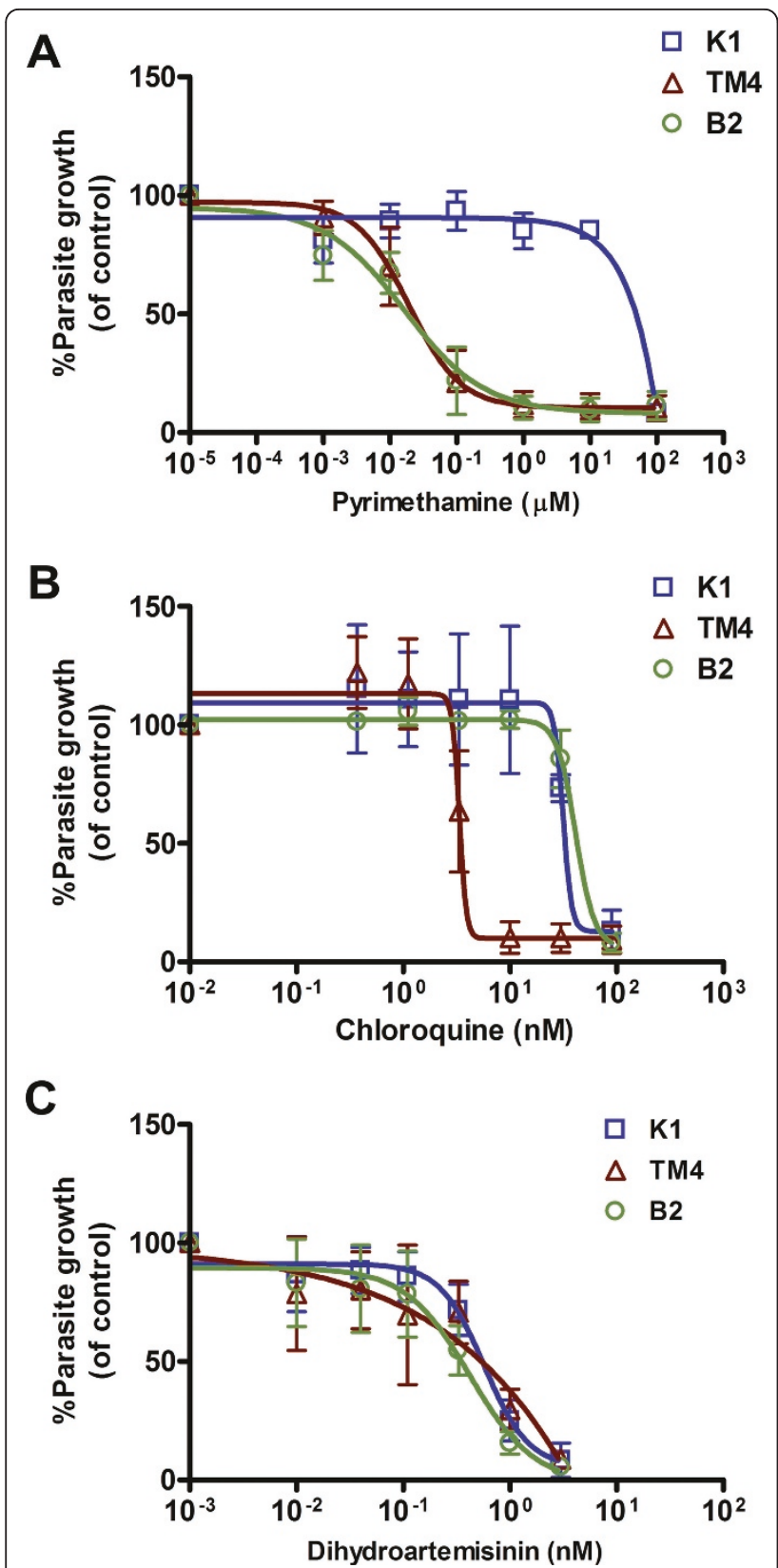

Figure 3 Sensitivity of transgenic Plasmodium falciparum expressing wild-type PvDHFR-TS enzyme to pyrimethamine (A), chloroquine (B) and dihydroartemisinin (C). The growth of parasites treated with pyrimethamine, chloroquine and dihydroartemisinin was detected using the SYBR Green I staining assay. The percentage of parasite growth was plotted against drug concentrations. Data were shown as mean \pm S.D. of at least 3 independent experiments. K1: P. falciparum K1CB1; TM4: P. falciparum TM4/8.2; B2: transgenic PfPvDTCIB2.

Drug sensitivity analysis of transgenic Plasmodium berghei stably expressing PvDHFR-TS enzymes

After inoculation, mice in the untreated control group showed a progressively increasing parasitaemia, and all the mice died by day 11 (data not shown). As shown in Figure 4A and Table 1, the transgenic PbPvDTcl4 demonstrated a drug susceptibility profile similar to that of the wild-type parental PbGFP with an $\mathrm{ED}_{50}$ of $0.53 \pm$ $0.24 \mathrm{mg} / \mathrm{kg}$ and $0.69 \pm 0.21 \mathrm{mg} / \mathrm{kg}$, respectively. This demonstrated that the wild-type PvDHFR-TS enzyme was equally susceptible to the antifolate compound compared with wild-type PbDHFR-TS. In contrast, transgenic PbPvSP21cl2 was approximately 40-fold more resistant to pyrimethamine than the $\mathrm{PbPvDTcl} 4$ parasite line (Figure 4A). Therefore, the double mutant $P$. vivax DHFR-TS confers a high level of resistance to pyrimethamine in P. berghei.

The transgenic $P$. berghei lines were also tested with the non-antifolate drugs chloroquine and artesunate. As shown in Figure 4B and Table 1, all parasite lines were similarly susceptible to chloroquine. The $\mathrm{ED}_{50}$ values against chloroquine were $1.56 \pm 0.12 \mathrm{mg} / \mathrm{kg}, 2.85 \pm 0.17$ $\mathrm{mg} / \mathrm{kg}$, and $3.88 \pm 0.13 \mathrm{mg} / \mathrm{kg} \mathrm{mg} / \mathrm{kg}$ in parental PbGFP, PbPvDTcl4 and PbPvSP21cl2 parasites, respectively. Artesunate is extremely potent against pyrimethamine-resistant parasites. It is a semi-synthetic derivative of artemisinin that is water-soluble and may therefore be given by injection. All parasite lines were also susceptible to artesunate treatment with $\mathrm{ED}_{50}$ values of $5.43 \pm$ $0.42 \mathrm{mg} / \mathrm{kg}, 7.43 \pm 0.30 \mathrm{mg} / \mathrm{kg}$ and $7.59 \pm 0.33 \mathrm{mg} / \mathrm{kg}$ in parental PbGFP, PbPvDTcl4 and PbPvSP21cl2 parasites, respectively (Figure and Table 1).

\section{Discussion and Conclusions}

This study describes the generation of both in vitro and in vivo transgenic Plasmodium parasites stably expressing $P$. vivax DHFR-TS enzyme and the application of these parasites for assessing antifolate compound efficacy. The transgenic Plasmodium parasites harbour only one copy of Pvdhfr-ts gene, which is under the control of the endogenous $d h f r$-ts regulatory sequences. The fact that it is possible to replace the endogenous Plasmodium dhfr-ts with a homologue from a different species suggests that $d h f r$-ts function is conserved among species of this genus. This is in agreement with biochemical evidence that cross-species heterodimers of Plasmodium DHFR-TS are fully functional [34]. While the expression of double mutant PvDHFR-TS SP21 was not deleterious and can support the growth of the transgenic $P$. berghei parasites, attempts to obtain P. falciparum stably expressing double mutant PvDHFR-TS SP21 were not successful (data not shown). The reasons may be the catalytic activity of PvDHFR-TS SP21 is insufficient to support the growth of transgenic $P$. falciparum. It was reported that the catalytic activity, as determined by $k_{\text {cat }} / K_{\mathrm{m}}$ of H2folate, of PvDHFR SP21 is approximately 13 times and 27 times lower than that of 
Table 1 Drug sensitivity of transgenic Plasmodium expressing PvDHFR-TS to standard anti-malarials

\begin{tabular}{|c|c|c|c|}
\hline \multirow[t]{2}{*}{ P. falciparum } & \multicolumn{3}{|c|}{$I C_{50} \pm$ S.D. } \\
\hline & Pyrimethamine $(\mu \mathrm{M})$ & Chloroquine (nM) & Dihydroartemisinin ( $\mathrm{nM}$ \\
\hline P. falciparum TM4/8.2 & $0.03 \pm 0.02$ & $4.3 \pm 1.4$ & $0.6 \pm 0.1$ \\
\hline P. falciparum K1CB1 & $28.7 \pm 1.7$ & $46.0 \pm 3.1$ & $0.7 \pm 0.3$ \\
\hline PfPvDTcIB2 & $0.03 \pm 0.02$ & $49.5 \pm 5.8$ & $0.4 \pm 0.1$ \\
\hline \multirow[t]{2}{*}{ P. berghei } & \multicolumn{3}{|c|}{$\mathrm{ED}_{50} \pm$ S.D. $(\mathrm{mg} / \mathrm{kg})$} \\
\hline & Pyrimethamine & Chloroquine & Artesunate \\
\hline P. berghei GFP & $0.69 \pm 0.21$ & $1.56 \pm 0.12$ & $5.43 \pm 0.42$ \\
\hline PbPvDTcl4 & $0.53 \pm 0.24$ & $2.85 \pm 0.17$ & $7.43 \pm 0.30$ \\
\hline PbPvSP21cl2 & $>30$ & $3.88 \pm 0.13$ & $7.59 \pm 0.33$ \\
\hline
\end{tabular}

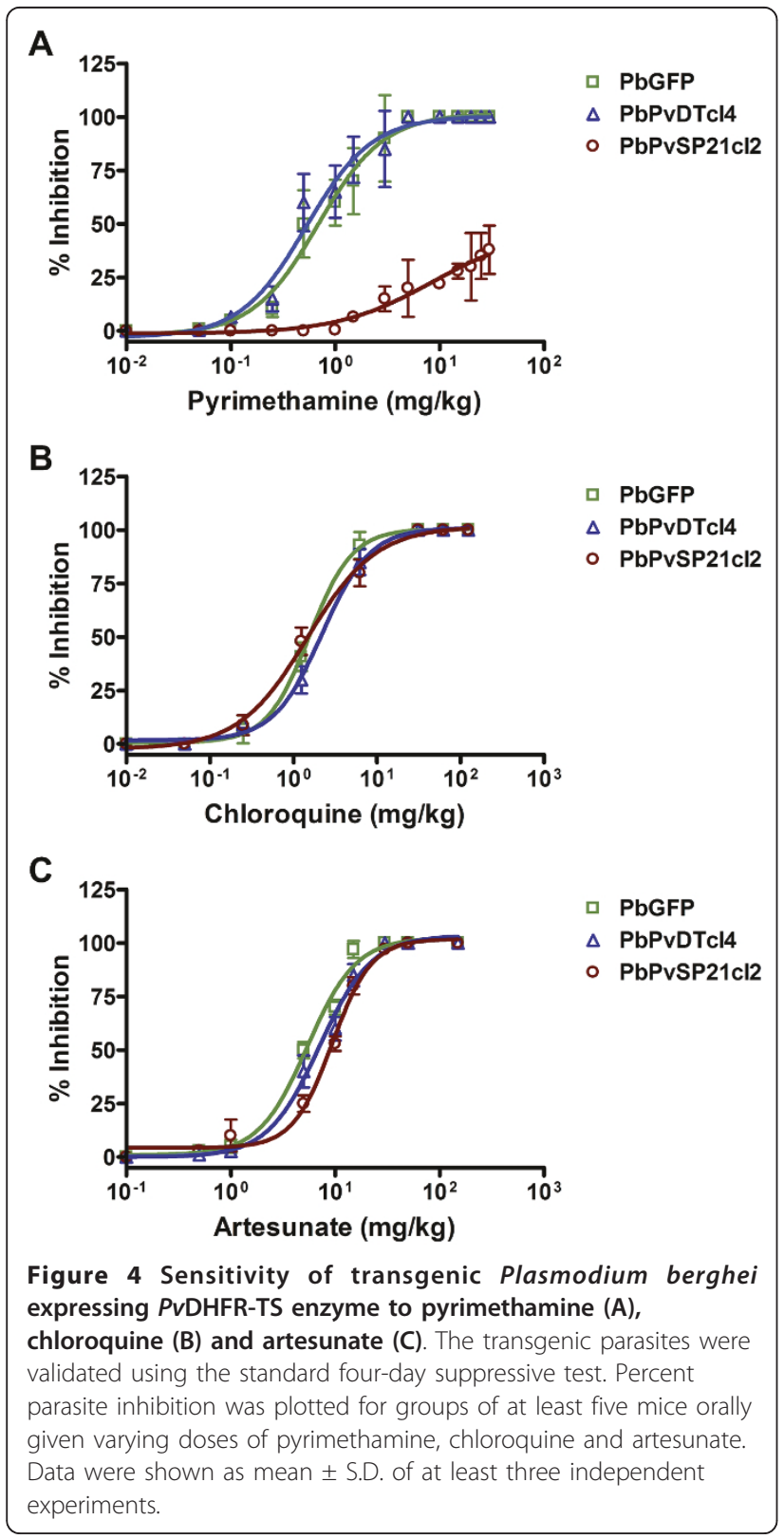

wild-type PvDHFR [11] and wild-type PfDHFR [35], respectively.

A major advantage of the Pvdhfr-ts transgenic parasites generated in the study is that the gene is stably maintained in an integrated fashion. This issue is important since it has been reported that the apparent copy number of plasmid DNA in episomally transfected Plasmodium is variable, which may affect the drug sensitivity profile in drug testing assays. The reasons for varying copy numbers are related to the nature of the episomal plasmid itself and the level and duration of drug pressure [36]; moreover, higher drug concentration are known to select for increased copy numbers of the episome [37]. In the absence of drug pressure, both transgenic $P$. falciparum and $P$. berghei lose episomal transgenes [36,38,39]; hence, constant drug pressure is needed to maintain the episomes. In the earlier report of P. falciparum episomally expressing $P v d h f r-t s$, no effect of episomal copy number on the drug sensitivity profile was observed, probably owing to the tight regulation of the parasite DHFR-TS expression level $[22,40]$. However, the episomal copy number did vary since the drug used to maintain the episome must be withdrawn for compound testing, hence the episomal system may not be sufficiently robust as a general drug screening method.

In this study, in vitro and in vivo Plasmodium models have been generated specifically for anti-PvDHFR screening. There are reports that compounds that have anti- $P$. falciparum activity in in vitro screening do not show the same anti-Plasmodial activity in in vivo models, such as $P$. berghei $[41,42]$. It is therefore premature to assume that compounds found to be active in vitro will also be efficacious in vivo before pharmacokinetic studies of the compounds in animal models have been conducted, since compounds with poor pharmacokinetic properties may simply not reach their targets. At present, the only in vivo model for $P$. vivax is $P$. cynomolgi infecting primates [43]. This model is expensive and 
restricted to specialized laboratories; hence the transgenic $P$. berghei expressing $P$. vivax targets is an attractive alternative experimental model.

In conclusion, transgenic $P$. falciparum and $P$. berghei stably expressing $P v$ DHFR-TS replacing the endogenous Plasmodium dhfr-ts were successfully generated as in vitro and in vivo Plasmodium drug screening models. The drug sensitivities of the transgenic lines varied according to the introduced $P v d h f r-t s$ gene. Until a routine continuous in vitro culture system is developed for $P$. vivax, these transgenic models will be useful for screening of anti-P. vivax compounds targeting the $P v$ DHFR enzyme. A similar approach could be used to generate transgenic models specific for other targets of interest, thus facilitating the development of anti- $P$. vivax drugs in general.

\section{Acknowledgements}

This work is within the objectives and framework of the TDR Transfection Network on "Application of Transfection Technology for Drug Screening and Immunological Studies in Tropical Parasitic Diseases" co-sponsored by the UNICEF/UNDP/World Bank/WHO Special Programme for Research and Training in Tropical Diseases (TDR) and Howard Hughes Medical Institute (HHMI, USA) to CU and SK. SK is an international research scholar of HHMI. VS acknowledges the Thailand Graduate Institute of Science and Technology (TGIST) for support. We thank Philip Shaw for his critical reading of the manuscript. We also wish to thank Andrew Waters and Chris Janse at Leiden University Medical Center, the Netherlands for valuable discussions and for providing the transgenic $P$. berghei parasite line MRA-867 and transfection vectors; Sodsri Thaithong at Chulalongkorn University, Thailand for P. falciparum strains TM4/8.2 and K1CB1; Ubolsree Leartsakulpanich, BIOTEC, Thailand for Pvdhfr-ts genes and Dafra Pharma International, Belgium for dihydroartemisinin.

\section{Author details}

${ }^{1}$ Protein-Ligand Engineering and Molecular Biology Laboratory, National Center for Genetic Engineering and Biotechnology (BIOTEC), National Science and Technology Development Agency (NSTDA), Thailand Science Park, Pathumthani 12120, Thailand. ${ }^{2}$ Department of Biochemistry, Faculty of Medicine, Chiang Mai University, Chiang Mai 50200, Thailand.

\section{Authors' contributions}

VS, CU, SS, YY and SK conceived and designed the project. VS, CU and PP performed experiments. VS and CU prepared the manuscript. SS, SK and YY critically reviewed the manuscript. All authors read and approved the final manuscript.

\section{Competing interests}

The authors declare that they have no competing interests.

Received: 17 August 2011 Accepted: 7 October 2011

Published: 7 October 2011

\section{References}

1. World Health Organization: World malaria report 2010. Geneva, Switzerland; 2010.

2. Carlton JM, Sina BJ, Adams JH: Why Is Plasmodium vivax a Neglected Tropical Disease? PLoS Negl Trop Dis 2011, 5(6):e1160

3. Schuurkamp GJ, Spicer PE, Kereu RK, Bulungol PK, Rieckmann KH: Chloroquine-resistant Plasmodium vivax in Papua New Guinea. Trans $R$ Soc Trop Med Hyg 1992, 86(2):121-122

4. Rieckmann $\mathrm{KH}$, Davis DR, Hutton DC: Plasmodium vivax resistance to chloroquine? Lancet 1989, 2(8673):1183-1184.

5. Baird JK: Chloroquine resistance in Plasmodium vivax. Antimicrob Agents Chemother 2004, 48(11):4075-4083.
6. Yuthavong Y: Basis for antifolate action and resistance in malaria. Microbes Infect 2002, 4(2):175-182.

7. Yuthavong Y, Yuvaniyama J, Chitnumsub P, Vanichtanankul J, Chusacultanachai S, Tarnchompoo B, Vilaivan T, Kamchonwongpaisan S: Malarial (Plasmodium falciparum) dihydrofolate reductase-thymidylate synthase: structural basis for antifolate resistance and development of effective inhibitors. Parasitology 2005, 130(Pt 3):249-259.

8. Cowman AF, Morry MJ, Biggs BA, Cross GA, Foote SJ: Amino acid changes linked to pyrimethamine resistance in the dihydrofolate reductasethymidylate synthase gene of Plasmodium falciparum. Proc Natl Acad Sci USA 1988, 85(23):9109-9113.

9. Sirawaraporn W, Prapunwattana P, Sirawaraporn R, Yuthavong Y, Santi DV: The dihydrofolate reductase domain of Plasmodium falciparum thymidylate synthase-dihydrofolate reductase. Gene synthesis, expression, and anti-folate-resistant mutants. J Biol Chem 1993, 268(29):21637-21644.

10. Wu Y, Kirkman LA, Wellems TE: Transformation of Plasmodium falciparum malaria parasites by homologous integration of plasmids that confer resistance to pyrimethamine. Proc Natl Acad Sci USA 1996, 93(3):1130-1134

11. Leartsakulpanich $U$, Imwong M, Pukrittayakamee S, White NJ, Snounou G, Sirawaraporn W, Yuthavong Y: Molecular characterization of dihydrofolate reductase in relation to antifolate resistance in Plasmodium vivax. $\mathrm{Mol}$ Biochem Parasitol 2002, 119(1):63-73.

12. Tahar R, de Pecoulas PE, Basco LK, Chiadmi M, Mazabraud A: Kinetic properties of dihydrofolate reductase from wild-type and mutant Plasmodium vivax expressed in Escherichia coli. Mol Biochem Parasitol 2001, 113(2):241-249.

13. Imwong M, Pukrittakayamee S, Looareesuwan S, Pasvol G, Poirreiz J, White NJ, Snounou G: Association of genetic mutations in Plasmodium vivax dhfr with resistance to sulfadoxine-pyrimethamine: geographical and clinical correlates. Antimicrob Agents Chemother 2001, 45(11):3122-3127.

14. Yuvaniyama J, Chitnumsub P, Kamchonwongpaisan S, Vanichtanankul J, Sirawaraporn W, Taylor P, Walkinshaw MD, Yuthavong Y: Insights into antifolate resistance from malarial DHFR-TS structures. Nat Struct Biol 2003, 10(5):357-365.

15. Kongsaeree $P$, Khongsuk $P$, Leartsakulpanich $U$, Chitnumsub $P$, Tarnchompoo B, Walkinshaw MD, Yuthavong Y: Crystal structure of dihydrofolate reductase from Plasmodium vivax: pyrimethamine displacement linked with mutation-induced resistance. Proc Natl Acad Sci USA 2005, 102(37):13046-13051.

16. Chotivanich K, Silamut K, Udomsangpetch R, Stepniewska KA Pukrittayakamee S, Looareesuwan S, White NJ: Ex-vivo short-term culture and developmental assessment of Plasmodium vivax. Trans $R$ Soc Trop Med Hyg 2001, 95(6):677-680.

17. Russell BM, Udomsangpetch R, Rieckmann $\mathrm{KH}$, Kotecka BM, Coleman RE, Sattabongkot J: Simple in vitro assay for determining the sensitivity of Plasmodium vivax isolates from fresh human blood to antimalarials in areas where P. vivax is endemic. Antimicrob Agents Chemother 2003, 47(1):170-173

18. Panichakul T, Sattabongkot J, Chotivanich K, Sirichaisinthop J, Cui L, Udomsangpetch R: Production of erythropoietic cells in vitro for continuous culture of Plasmodium vivax. Int J Parasitol 2007, 37(14):1551-1557.

19. Hastings MD, Sibley $\mathrm{CH}$ : Pyrimethamine and WR99210 exert opposing selection on dihydrofolate reductase from Plasmodium vivax. Proc Natl Acad Sci USA 2002, 99(20):13137-13141.

20. Bunyarataphan S, Leartsakulpanich U, Taweechai S, Tarnchompoo B, Kamchonwongpaisan S, Yuthavong Y: Evaluation of the activities of pyrimethamine analogs against Plasmodium vivax and Plasmodium falciparum dihydrofolate reductase-thymidylate synthase using in vitro enzyme inhibition and bacterial complementation assays. Antimicrob Agents Chemother 2006, 50(11):3631-3637.

21. O'Neil MT, Korsinczky ML, Gresty KJ, Auliff A, Cheng Q: A nove Plasmodium falciparum expression system for assessing antifolate resistance caused by mutant $P$. vivax dihydrofolate reductasethymidylate synthase. J Infect Dis 2007, 196(3):467-474

22. Auliff AM, Adams JH, O'Neil MT, Cheng Q: Defining the role of mutations in Plasmodium vivax dihydrofolate reductase-thymidylate synthase gene 
using an episomal Plasmodium falciparum transfection system Antimicrob Agents Chemother 2010, 54(9):3927-3932.

23. Trager W, Jensen JB: Human malaria parasites in continuous culture. Science 1976, 193(4254):673-675.

24. Franke-Fayard B, Trueman H, Ramesar J, Mendoza J, van der Keur M, van der Linden R, Sinden RE, Waters AP, Janse CJ: A Plasmodium berghei reference line that constitutively expresses GFP at a high level throughout the complete life cycle. Mol Biochem Parasitol 2004, 137(1):23-33.

25. Janse CJ, Franke-Fayard B, Waters AP: Selection by flow-sorting of genetically transformed, GFP-expressing blood stages of the rodent malaria parasite, Plasmodium berghei. Nat Protoc 2006, 1(2):614-623.

26. Mamoun CB, Gluzman IY, Goyard S, Beverley SM, Goldberg DE: A set of independent selectable markers for transfection of the human malaria parasite Plasmodium falciparum. Proc Natl Acad Sci USA 1999, 96(15):8716-8720.

27. Maier AG, Braks JA, Waters AP, Cowman AF: Negative selection using yeast cytosine deaminase/uracil phosphoribosyl transferase in Plasmodium falciparum for targeted gene deletion by double crossover recombination. Mol Biochem Parasitol 2006, 150(1):118-121.

28. Braks JA, Franke-Fayard B, Kroeze H, Janse CJ, Waters AP: Development and application of a positive-negative selectable marker system for use in reverse genetics in Plasmodium. Nucleic Acids Res 2006, 34(5):e39.

29. Crabb BS, Triglia T, Waterkeyn JG, Cowman AF: Stable transgene expression in Plasmodium falciparum. Mol Biochem Parasitol 1997, 90(1):131-144

30. Janse CJ, Ramesar J, Waters AP: High-efficiency transfection and drug selection of genetically transformed blood stages of the rodent malaria parasite Plasmodium berghei. Nat Protoc 2006, 1(1):346-356.

31. Waters AP, Thomas AW, van Dijk MR, Janse CJ: Transfection of malaria parasites. Methods 1997, 13(2):134-147.

32. Izumiyama S, Omura M, Takasaki T, Ohmae H, Asahi H: Plasmodium falciparum: development and validation of a measure of intraerythrocytic growth using SYBR Green I in a flow cytometer. Exp Parasitol 2009, 121(2):144-150.

33. Peters W: The chemotherapy of rodent malaria, XXII. The value of drugresistant strains of $P$. berghei in screening for blood schizontocidal activity. Ann Trop Med Parasitol 1975, 69(2):155-171.

34. Chanama M, Chanama S, Shaw PJ, Chitnumsub P, Leartsakulpanich U, Yuthavong Y: Formation of catalytically active cross-species heterodimers of thymidylate synthase from Plasmodium falciparum and Plasmodium vivax. Mol Biol Rep 2011, 38(2):1029-1037.

35. Sirawaraporn W, Sathitkul T, Sirawaraporn R, Yuthavong Y, Santi DV: Antifolate-resistant mutants of Plasmodium falciparum dihydrofolate reductase. Proc Natl Acad Sci USA 1997, 94(4):1124-1129.

36. de Koning-Ward TF, Fidock DA, Thathy V, Menard R, van Spaendonk RM, Waters AP, Janse CJ: The selectable marker human dihydrofolate reductase enables sequential genetic manipulation of the Plasmodium berghei genome. Mol Biochem Parasitol 2000, 106(2):199-212.

37. Epp C, Raskolnikov D, Deitsch KW: A regulatable transgene expression system for cultured Plasmodium falciparum parasites. Malar J 2008, 7:86.

38. Waterkeyn JG, Crabb BS, Cowman AF: Transfection of the human malaria parasite Plasmodium falciparum. Int J Parasitol 1999, 29(6):945-955.

39. van Dijk MR, Vinkenoog R, Ramesar J, Vervenne RA, Waters AP, Janse CJ: Replication, expression and segregation of plasmid-borne DNA in genetically transformed malaria parasites. Mol Biochem Parasitol 1997, 86(2):155-162.

40. Zhang K, Rathod PK: Divergent regulation of dihydrofolate reductase between malaria parasite and human host. Science 2002, 296(5567):545-547.

41. Gutteridge CE, Nichols DA, Curtis SM, Thota DS, Vo JV, Gerena L, Montip G, Asher CO, Diaz DS, Ditusa CA, et al: In vitro and in vivo efficacy and in vitro metabolism of 1-phenyl-3-aryl-2-propen-1-ones against Plasmodium falciparum. Bioorg Med Chem Lett 2006, 16(21):5682-5686.

42. Steele JC, Warhurst DC, Kirby GC, Simmonds MS: In vitro and in vivo evaluation of betulinic acid as an antimalarial. Phytother Res 1999, 13(2):115-119.

43. Kocken $\mathrm{CH}$, Remarque EJ, Dubbeld MA, Wein S, van der Wel A, Verburgh RJ, Vial HJ, Thomas AW: Statistical model to evaluate in vivo activities of antimalarial drugs in a Plasmodium cynomolgi-macaque model for
Plasmodium vivax malaria. Antimicrob Agents Chemother 2009, 53(2):421-427.

doi:10.1186/1475-2875-10-291

Cite this article as: Somsak et al: Transgenic Plasmodium parasites stably expressing Plasmodium vivax dihydrofolate reductase-thymidylate synthase as in vitro and in vivo models for antifolate screening. Malaria Journal 2011 10:291.

\section{Submit your next manuscript to BioMed Central and take full advantage of:}

- Convenient online submission

- Thorough peer review

- No space constraints or color figure charges

- Immediate publication on acceptance

- Inclusion in PubMed, CAS, Scopus and Google Scholar

- Research which is freely available for redistribution

Submit your manuscript at www.biomedcentral com/submit
C Biomed Central 